\title{
The clinical spectrum of inflammatory-angiopathic neuropathy
}

\author{
YADOLLAH HARATI, ENAYAT NIAKAN \\ From the Department of Neurology, Baylor College of Medicine and Veterans Administration Medical Center, \\ Houston, Texas, USA
}

SUMMARY Thirty-three patients with inflammatory-angiopathic neuropathy diagnosed by sural nerve biopsy, were investigated to determine the underlying disease. Twenty-six patients had symmetrical sensorimotor polyneuropathy and seven had mononeuropathy multiplex. An aetiology for inflammatory-angiopathic neuropathy was found in only eight patients: typical collagen vascular disease in five and malignant tumour in three. Sixteen patients received prednisone and/or immunosuppressive drug therapy and $12(75 \%)$ responded to treatment. This study demonstrates that typical collagen vascular diseases are not the most common cause of inflammatory-angiopathic neuropathy, that symmetrical polyneuropathy is seen in $75.8 \%$ of inflammatory-angiopathic neuropathy patients. Prednisone and/or immunosuppressive agents appear effective regardless of aetiology.

Inflammatory-angiopathic neuropathy is often associated with collagen vascular diseases, ${ }^{1-9}$ with mononeuropathy multiplex being the most common clinical manifestation. ${ }^{10}$ Conversely, the incidence of collagen vascular disease and mononeuropathy multiplex among patients with sural nerve biopsy proven inflammatory-angiopathic neuropathy has not been established. This study examines the underlying disease in 33 patients with inflammatory-angiopathic neuropathy, diagnosed by sural nerve biopsy.

\section{Methods and patients}

Thirty-three of 250 consecutive sural nerve biopsies performed at our institution or submitted to us by other hospitals or physicians demonstrated inflammatory-angiopathic neuropathy. Each patient's record was examined. There were 15 females and 18 males ranging in age from 14 to 75 (mean, 54) years, with duration of their clinical neuropathy from 1 to 72 (mean, 18.5) months. All patients had undergone total sural nerve biopsy.

A portion of nerve was frozen and transverse and longi-

Address for reprint requests: Yadollah Harati, MD, Department of Neurology, Baylor College of Medicine, 6501 Fannin, Houston, Texas 77030 , USA

Received 26 November 1985 and in revised form 26 January 1986. Accepted 1 March 1986. tudinal sections stained with modified Gomori trichrome (TRI), haematoxylin and eosin, cresyl violet and periodic acid schiff (PAS). Other portions of nerve were fixed in glutaraldehyde, underwent osmification, and were teased.

The criteria for diagnosis of inflammatory-angiopathic neuropathy on sural nerve biopsy were similar to those established by Conn and Dyck ${ }^{2}$ and Cupps and Fauci: ${ }^{3}$ perivascular and intramural infiltration of inflammatory cells with necrosis of the blood vessel wall; and/or chronic arterial changes (combination of endothelial proliferation, perivascular fibrosis and fibrosis of the arterial, and narrowed or occluded lumen), indicative of previous injury to arterial wall (fig).

Nerve biopsy specimens with evidence of perivascular infiltration of inflammatory cells, but without angiopathy were excluded.

Teased nerve pathology was classified into three groups: (1) demyelination/remyelination; (2) predominantly axonal degeneration; or (3) predominantly demyelination/ remyelination with some axonal degeneration. Axonal degeneration was identified as "myelin digestion chamber" on TRI stain, and "myelin ovoids and balls" in teased nerve fibres.

\section{Results}

Most of the 33 patients studied had a chronic course, with duration of greater than 6 months. Based on clinical examination, electromyography (EMG) and nerve conduction velocities (NCV); twenty-five $(75 \cdot 8 \%)$ had diffuse, distal sensorimotor poly- 


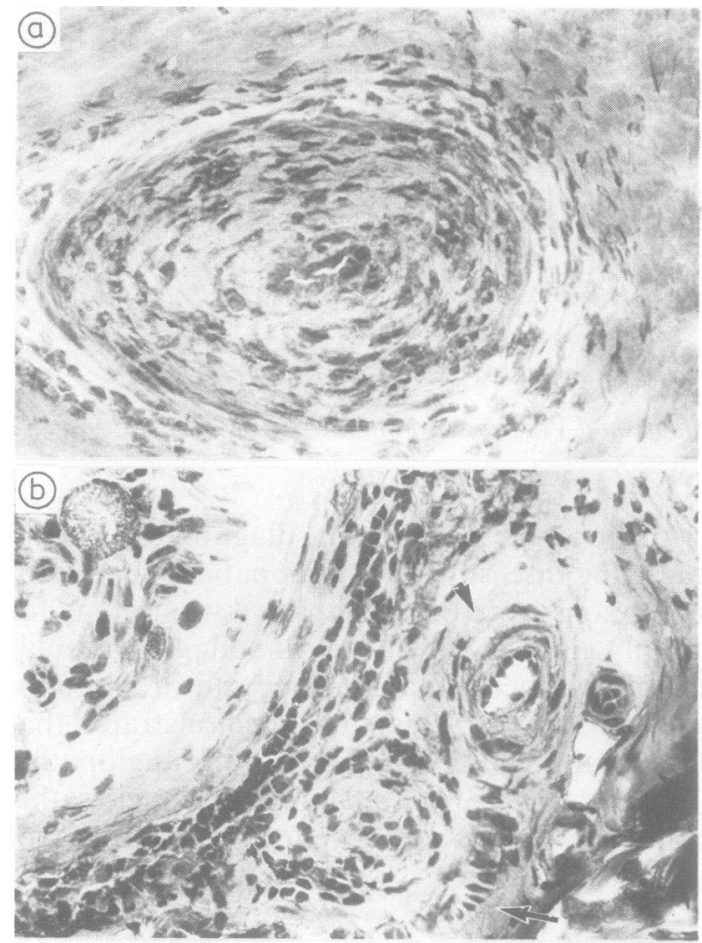

Fig (A) Marked intimal proliferation, narrowed lumen, mononuclear cell infiltrations around blood vessels and intramural, and necrosis in vessels walls $\times 500$.

$(B)$, perivascular and intramural infiltration of mononuclear cells, also inflammatory cells extending to the perineurium; note one blood vessel with occluded lumen (arrow) and other with thick wall (arrowhead) $\times 450$.

neuropathy; eight (24.2\%) demonstrated mononeuropathy multiplex. Mononeuropathy multiplex was defined when clinical examination and/or NCV showed asymmetrical sensorimotor peripheral neuropathy or when an asymmetrical neuropathy progressed to a symmetrical neuropathy during the course of illness (confluent mononeuropathy multi- plex). Extensive clinical and laboratory evaluation including tests for collagen vascular disease revealed an aetiology for inflammatory-angiopathic neuropathy in only eight patients: rheumatoid arthritis (2), polyarteritis nodosa (2), scleroderma (1), and malignant tumour (3). Malignancies included carcinoma of the cervix (1), both lymphoma and prostatic carcinoma (1), and histiocytic lymphoma (1). Diagnosis of collagen vascular disease was made by using criteria established by The American Rheumatism Association. ${ }^{11}$ Of the 25 cases with no known aetiology, four had some clinical and/or serological abnormalities suggestive of collagen vascular disease, but not diagnostic for a specific disease (these patients were defined as atypical collagen vascular disease); six other patients had elevated cerebrospinal fluid protein. In no patient were cryoglobulinaemia or similar disorders identified.

Teased nerve fibres revealed segmental demyelination/remyelination in two cases, predominantly axonal degeneration in 13, and predominantly segmental demyelination/remyelination with some axonal degeneration in 18 .

The severity of the clinical neuropathy correlated with the severity of myelinated fibre reduction in the biopsy specimen, utilising photomicrographs of transverse sections, compared with normal control.

Sixteen patients received prednisone and/or immunosuppressive agents (cylophosphamide or azathioprine) in varying doses for variable periods. Twelve $(75 \%)$ patients responded to treatment, regardless of the aetiology of their inflammatory-angiopathic neuropathy (table 1). The degree of improvement was from slight to complete, as judged by the clinical and electrophysiological evaluations.

\section{Discussion}

The association of inflammatory-angiopathic neuropathy with collagen vascular disease has been well documented, ${ }^{1-9}$ and it is the general opinion that col-

Table 1 Clinical diagnosis and response to treatment

\begin{tabular}{llll}
\hline No of patients & Diagnosis & Response to treatment \\
\cline { 3 - 4 } & & Prednisone & Prednisone and immunosuppressive \\
\hline 1 & Rheumatoid arthritis & Yes & No (1)† \\
2 & Polyarteritis nodosa & Yes & Yes (1) \\
1 & Atypical vascular disease & No & Yes (2) \\
6 & CPN & Yes (4) & Yes (3)* \\
\hline
\end{tabular}

( ): number of patients.

*Three did not respond to prednisone and did not receive immunosuppressive drugs.

†Patient did not respond to treatment and died due to necrotising vasculitis involving gastrointestinal systems.

$\mathrm{CPN}$ : chronic progressive neuropathy with elevated spinal fluid protein. 
Table 2 Relationship between clinical diagnosis and type of neuropathy and pathological findings

\begin{tabular}{|c|c|c|c|c|c|c|}
\hline \multirow{2}{*}{$\begin{array}{l}\text { No of } \\
\text { patients }\end{array}$} & \multirow[t]{2}{*}{ Diagnosis } & \multicolumn{2}{|c|}{ Type of neuropathy } & \multicolumn{3}{|c|}{ Teased nerve findings } \\
\hline & & Polyneuropathy & $\begin{array}{l}\text { Mononeuropathy } \\
\text { multiplex }\end{array}$ & $S D / R M^{*}$ & $\begin{array}{l}S D / R M / \\
\text { Axo' }\end{array}$ & $\begin{array}{l}\text { Axonal } \\
\text { Degeneration }\end{array}$ \\
\hline 5 & Collagen vascular & & & & & \\
\hline & disease & 4 & 1 & 0 & 2 & 3 \\
\hline $\begin{array}{l}3 \\
4\end{array}$ & $\begin{array}{l}\text { Neoplasm } \\
\text { Atypical collagen }\end{array}$ & 3 & 0 & 0 & 2 & 1 \\
\hline & vascular disease & 2 & 2 & 1 & 2 & 1 \\
\hline 6 & CPN & 4 & 2 & 0 & 4 & 2 \\
\hline 15 & Unknown & 12 & 3 & 1 & 8 & 6 \\
\hline
\end{tabular}

*SD/RM: Segmental demyelination/remyelination.

†SD/RM/Axo: Segmental demyelination/remyelination and axonal degeneration.

lagen vascular disease is the most common cause of inflammatory-angiopathic neuropathy. Recently, Kissel et al reported that of 16 patients with neuropathy and necrotising vasculitis, only four $(25 \%)$ had a known collagen vascular disease. ${ }^{12}$

Most patients in our study did not receive a definitive diagnosis despite extensive investigation (table 2). Known collagen vascular diseases were found in only five $(15 \%)$ of our patients, and another four $(12 \%)$ had evidence to support a diagnosis of atypical collagen vascular disease. This low incidence of collagen vascular disease among our patients is likely due to the fact that we studied the clinical spectrum of patients with inflammatory-angiopathic neuropathy, rather than studying only patients with inflammatory-angiopathic neuropathy due to collagen vascular disease as have previous authors. ${ }^{4-9}$ However, the possibility that some of the patients with idiopathic inflammatory-angiopathic neuropathy, especially those who presented with atypical collagen vascular disease, may develop a collagen vascular disease later cannot be ruled out.

Cerebrospinal fluid protein was elevated in six of 25 patients $(24 \%)$ in whom no definitive diagnosis could be made. All six of these patients had a chronic progressive course and responded to prednisone and/or immuno-suppressive therapy. Elevation of cerebrospinal fluid protein usually is seen in inflammatory polyradiculoneuropathy. ${ }^{13}$ However, this diagnosis was excluded in our patients, based on clinical presentation, electrophysiological evaluation, and presence of angiopathic changes on sural nerve biopsy.

Three of our patients with inflammatoryangiopathic neuropathy had underlying malignancy, with their neuropathy being noted at the time, or within a few months, of the diagnosis of malignancy. The possibility that angiopathy was a coincidental finding in these patients cannot be excluded; however, inflammatory-angiopathic neuropathy has been reported as a remote effect of carcinoma or malignant tumour. ${ }^{14}$ Immune complexes may play a role in the pathophysiology of the angiopathy seen with carcinomas or reticuloendothelial tumours. ${ }^{3}$

The majority $(75.8 \%)$ of patients in our series presented with chronic progressive distal symmetrical sensorimotor polyneuropathy. Kissel et al reported a high incidence of symmetrical polyneuropathy in patients with necrotising vasculitis. ${ }^{12}$ Wee et al reported that 16 of 17 patients with inflammatory-angiopathic neuropathy had polyneuropathy, while one had mononeuropathy multiplex. ${ }^{9}$ Symmetrical polyneuropathy has also been reported in a high proportion of patients with inflammatory-angiopathic neuropathy due to rheumatoid arthritis. ${ }^{256}$ The discrepancy between these studies and the current belief that mononeuropathy multiplex is the hallmark of inflammatoryangiopathic neuropathy is not understood. It has been suggested that some patients may present with a clinical picture resembling mononeuropathy multiplex, but, have indeed, a polyneuropathy; and often symmetrical neuropathy is detected with adequate electrophysiologic testing. ${ }^{9}$ In our series, however, we considered patients to have mononeuropathy multiplex when they had a history of acute or subacute asymmetric sensorimotor peripheral neuropathy, despite diffusely abnormal nerve conduction velocities in some patients. Another explanation for the discrepancy may be that symmetrical polyneuropathy develops at a late stage of disease due to multiple nerve lesions. It is also possible that multifocal lesions summate, thereby producing symmetrical polyneuropathy, similar to that reported by Sugimura et $a l$, in two diabetic patients. ${ }^{15}$

The exact value of sural nerve biopsy in the detection of inflammatory-angiopathic neuropathy in patients with idiopathic mononeuropathy multiplex or symmetrical peripheral neuropathy is not known. Gareth et al studied nerve biopsies of 22 patients with mononeuropathy multiplex and confirmed only three cases of vasculitis, although six patients had a clinical 
diagnosis of collagen vascular disease. ${ }^{16}$ Dyck et al, in an intensive evaluation of patients with unclassified neuropathy, found that $21 \%$ had inflammatory neuropathy. ${ }^{17}$ In our series, only 33 of $250(13 \cdot 2 \%)$ sural nerve biopsies revealed inflammatory-angiopathic neuropathy. In most of these patients, nerve biopsy was performed only when an aetiology for their peripheral neuropathy was not known.

The finding of inflammatory-angiopathic neuropathy on sural nerve biopsy was helpful in the management of our patients. Seventy-five per cent of those receiving prednisone and/or immunosuppressive agents improved (table 1). Response was most pronounced in patients with elevated spinal fluid protein.

The type of pathologic abnormalities on teased nerve fibre preparation was not helpful in distinguishing different aetiologies of inflammatory-angiopathic neuropathy in our series (table 2). Most of our patients had predominantly segmental demyelination/remyelination with some axonal degeneration or predominantly axonal degeneration. Angiopathy can produce both segmental demyelination and axonal degeneration, depending on the severity of the ischaemia. ${ }^{4}$

In summary, this study demonstrates that typical collagen vascular diseases are not the most common cause of inflàmmatory-angiopathic neuropathy; indeed, most of the patients with inflammatoryangiopathic neuropathy did not have a determinable aetiology. Additionally, symmetrical distal sensorimotor polyneuropathy is seen in a high proportion of patients with inflammatory-angiopathic neuropathy. The presence of inflammatory-angiopathic neuropathy shown in biopsy specimens may significantly aid in the management of these patients, as they may respond to prednisone and/or immunosuppressive drug therapy, regardless of aetiology.

\section{References}

${ }^{1}$ Asbury AK, Johnson PC. Pathology of Peripheral Nerve.
London: WB Saunders Company, 1978.

${ }^{2}$ Conn DL, Dyck PJ. Angiopathic neuropathy in connective tissue disease. In: Dyck PJ, Thomas PK, Lambert EH, eds. Peripheral Neuropathy, Vol 2. Philadelphia: WB Saunders Company, 1984:2027-43.

${ }^{3}$ Cupps TR, Fauci AS. The Vasculitides. London: WB Saunders Company, 1981.

${ }^{4}$ Dyck PJ, Conn DL, Okazaki H. Necrotizing angiopathic neuropathy, three-dimensional morphology of fiber degeneration related to sites of occluded vessels. Mayo Clin Proc 1972;47:461-75.

${ }^{5}$ Hart FD, Golding JR, Mackenzie DH. Neuropathy in rheumatoid disease. Ann Rheum Dis 1957;16:471-80.

${ }^{6}$ Johnson RL, Smyth CT, Holt GW, et al. Steroid therapy and vascular lesions in rheumatoid arthritis. Arthritis Rheum 1955;2:224-49.

${ }^{7}$ Johnson RT, Richardson EP. The neurological manifestations of systemic lupus erythematosus: a clinicalpathological study of 24 cases of review of literature. Medicine 1968:47:337-69.

${ }^{8}$ Lovlace RE. Mononeuritis multiplex in polyarteritis nodosa. Neurology 1964;14:434-42.

${ }^{9}$ Wee SJ, Sunwood L, Oh SJ. Sural nerve biopsy in systemic necrotizing vasculitis. Am J Med 1981;71:525-32.

${ }^{10}$ Chany RW, Bell CL, Hallet M. Clinical characteristics and prognosis of vasculitic mononeuropathy multiplex. Arch Neurol 1984;41:618-21.

${ }^{11}$ Kelley WN, Harris ED, Ruddy S, Sledge CB. Textbook of Rheumatology. London: WB Saunders Company, 1985.

${ }^{12}$ Kissel JT, Slivka AP, Warmolts TR, Mendell JR. The clinical spectrum of necrotizing angiopathy of the peripheral nervous system. Ann Neurol 1985;18:251-7.

${ }^{13}$ Dyck PJ, Lais AC, Ohta M, et al. Chronic inflammatory polyradiculoneuropathy. Mayo Clin Proc 1975;50: 621-37.

${ }^{14}$ Johnson PC, Rolak LA, Hamilton RH, et al. Paraneoplastic vasculitis of nerve: a remote effect of cancer. Ann Neurol 1979;5:437-44.

${ }^{15}$ Sugimura K, Dyck PJ. Multifocal fiber loss in proximal sciatic nerve in symmetrical distal diabetic neuropathy. J Neurol Sci 1982;53:501-9.

${ }^{16}$ Gareth JP, Brown MJ, Asbury AK. Diagnostic value of nerve biopsy in mononeuritis multiplex. Neurology 1981;31:129-30.

${ }^{17}$ Dyck PJ, Oviatt KF, Lambert EH. Intensive evaluation of referred unclassified neuropathy yields improved diagnosis. Ann Neurol 1981;10:222-6. 